\title{
Primary Care Physician Referral to Physical Therapy for Musculoskeletal Conditions, 2003-2014
}

\author{
Janet K. Freburger, PT, $P h D^{1,2}$, Samannaaz Khoja, $P T, P h D^{7}$, and Timothy S. Carey, MD, MPH ${ }^{2,3}$ \\ 'Department of Physical Therapy, School of Health and Rehabilitation Science, University of Pittsburgh, Pittsburgh, PA, USA; ${ }^{2}$ Cecil G. Sheps Center \\ for Health Services Research, University of North Carolina, Chapel Hill, NC, USA; ${ }^{3}$ Departments of Medicine and Social Medicine, University of North \\ Carolina, Chapel Hill, NC, USA.
}

KEY WORDS: rehabilitation; access to care; workforce; musculoskeletal conditions; consultation.

J Gen Intern Med 33(6):801-3

DOI: $10.1007 / \mathrm{s} 11606-018-4426-6$

(c) Society of General Internal Medicine 2018

\section{INTRODUCTION}

Musculoskeletal conditions are a significant burden in the USA affecting more than one-half of adults and threequarters of those 65 years and older, making these conditions more common than hypertension, diabetes, and cardiovascular disease. ${ }^{1}$ Primary care physicians (PCPs) are typically the first point of contact for individuals seeking care for musculoskeletal conditions. ${ }^{2}$ With the development of new healthcare delivery and payment models and the increasing medical complexity of the primary care patient, PCPs are facing greater pressure to deliver coordinated, integrated, team-based care to achieve improved population health. ${ }^{3}$ While physical therapists are sometimes mentioned in team-based models of primary care, little empirical data are available on the extent to which PCPs engage physical therapists in patient care. Current guidelines recommend physical therapy (PT) as an essential component of first-line treatment for many musculoskeletal conditions.

We examined temporal trends in PCP referral to PT for musculoskeletal conditions and compared these trends to PCP referral to specialty physicians.

\section{METHODS}

We used 12 years of data (2003-2014) from the National Ambulatory Medical Care Survey, a national probability sample survey of office-based physicians engaged in patient care. Data collected on sampled visits include patient characteristics; visit characteristics; physician diagnosis; services/tests ordered (including referral to PT and/or another physician); and treatments/medications prescribed. Data on physician specialty are also collected.

We limited our analysis to visits to PCPs (i.e., general/ family practice, internal medicine, pediatrics, obstetrics/

Published online April 5, 2018 gynecology) for musculoskeletal conditions based on ICD-9$\mathrm{CM}$ codes and created the following diagnostic subgroups: arthropathies and related disorders (710-719); spine disorders (720-724, 846, 847); soft tissue disorders (725-729); osteopathies, chondropathies, musculoskeletal deformities (730739); fractures/dislocations (820-829, 830-839); extremity sprains/strains (840-845, 848); contusions (922-924). In addition to PT and physician referral data, we extracted data on patient characteristics (age, sex, race, ethnicity, insurance status) and the major reason for visit (i.e., acute problem, chronic problem, pre- or post-surgery, preventive).

\section{Analyses}

Analyses were conducted using Stata (14.2) and accounting for the multistage survey design. ${ }^{4}$ We pooled the data over 2-year intervals to increase estimate precision. ${ }^{5}$ To account for potential changes in the characteristics of ambulatory care visits over time, we estimated a multivariate logistic regression model with PT referral as the outcome; the 2-year intervals as categorical, explanatory variables; and age, sex, race, ethnicity, insurance, major reason for visit, and diagnostic subgroups as our control variables. We then calculated average adjusted prevalence rates for each 2-year period and assessed for a linear trend over time. ${ }^{6}$ We also estimated number of visits referred. We used the same approach to estimate referral rates and number of visits referred to specialty physicians. We conducted our analyses for the entire sample (i.e., all musculoskeletal-related ambulatory care visits) and for the three most prevalent diagnostic subgroups: arthropathies (32.3\% of all musculoskeletal visits), spine (28.3\%), and soft tissue disorders (17.9\%).

\section{RESULTS}

Figure 1 presents the adjusted rates of PT referral over time. Rates of referral for all musculoskeletal-related visits ranged from 94.4/1000 visits in 2003-2004 to 42.9/1000 visits in 2013-2014, a drop of over 50\%. The weighted number of visits referred ranged from 13.9 million visits in 2003-2004 to 5.1 million visits in 2013-2014. Rates of 


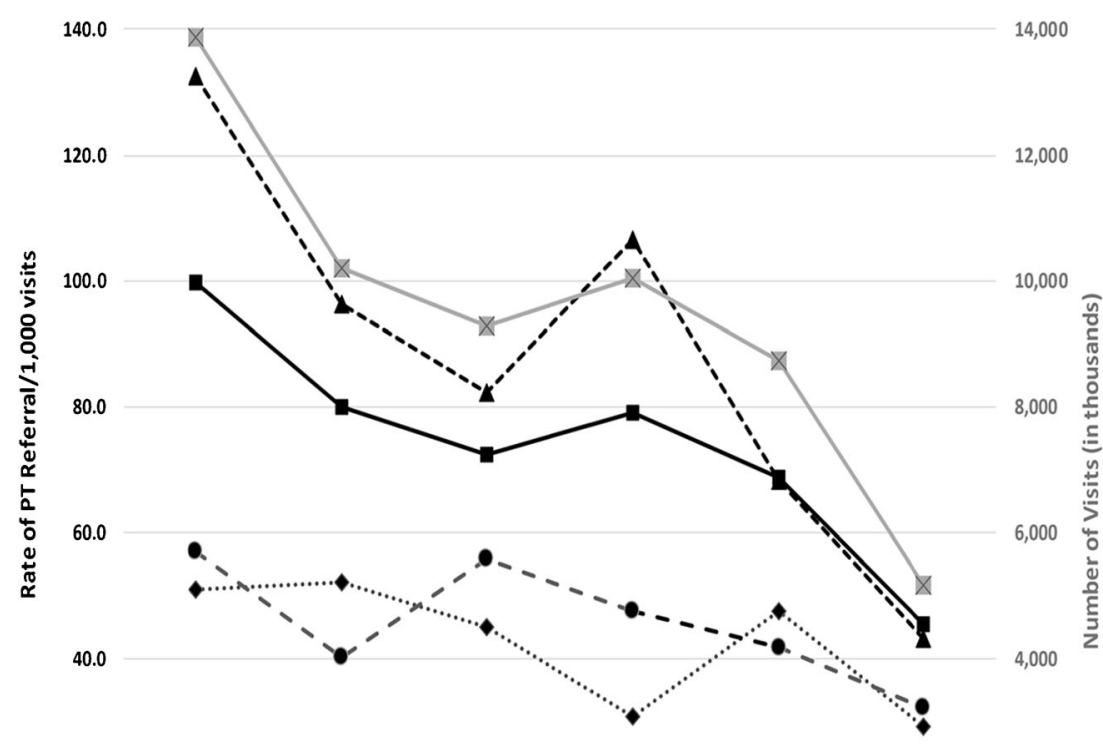

$20.0 \longrightarrow 2,000$

0.0

\begin{tabular}{|c|c|c|c|c|c|}
\hline 2003-4 & $2005-6$ & $2007-8$ & $2009-10$ & 2011-12 & 2013-14 \\
\hline \multicolumn{6}{|c|}{ 2-Year Intervals } \\
\hline
\end{tabular}

Fig. 1 Adjusted rates (Rates adjusted for age, race, sex, ethnicity, insurance, and diagnostic subgroup; MSK, musculoskeletal. MSK rate, linear trend test (coefficient $=-0.129, p=0.01$ ). Arthropathy rate, linear trend test (coefficient $=-0.101, p=0.06$ ). Soft tissue rate, linear trend test $($ coefficient $=-\mathbf{0 . 0 8 6}, p=0.07)$. Spine rate, linear trend test $($ coefficient $=-0.18, p=0.02)$ ) and number of musculoskeletal ambulatory care visits referred to physical therapy.

250.0

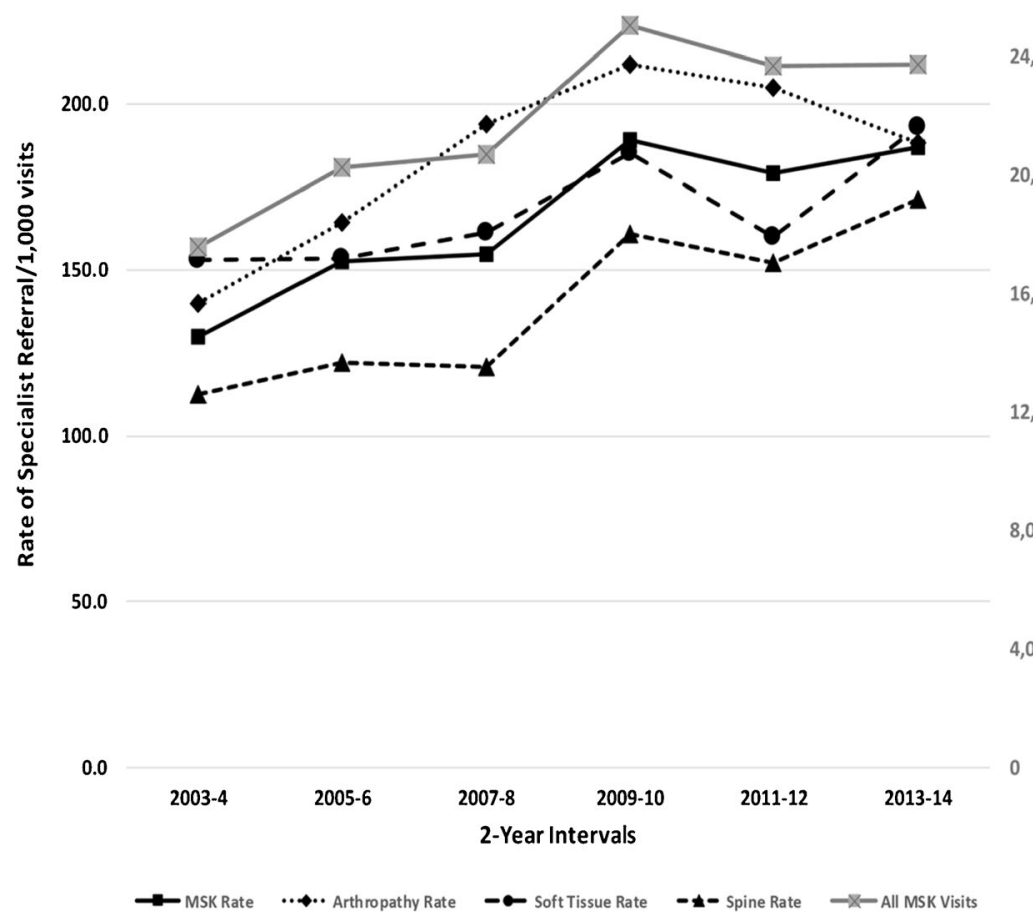

28,000

24,000

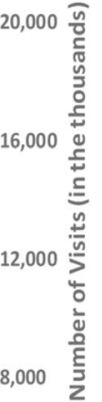

4,000

(1)

Fig. 2 Adjusted rates (Rates adjusted for age, race, sex, ethnicity, insurance, and diagnostic subgroup; MSK, musculoskeletal. MSK rate, linear trend test (coefficient $=0.086, p<.001)$. Arthropathy rate, linear trend test (coefficient $=0.073, p=0.007$ ). Soft tissue rate, linear trend test (coefficient $=0.050, p=0.14)$. Spine rate, linear trend test $($ coefficient $=0.100, p=0.001)$ ) and number of musculoskeletal ambulatory care visits referred to specialist physicians. 
referral followed similar trends for the diagnostic subgroups. While rates of PT referral decreased over time, rates of referrals to specialty physicians increased over the same period (Fig. 2).

\section{DISCUSSION}

We examined temporal trends in PT referral by PCPs and found referral rates declined between 2003 and 2014. Whether this decline represents more appropriate PT referral (i.e., decrease in inappropriate referrals) or missed opportunities (i.e., appropriate referrals not being made) is unclear. While PT referrals by primary care clinicians declined, referrals to specialist physicians increased over the same period. Had the decrease in PT referral rates reflected more judicious use of specialists by PCPs, we would have expected a similar trend for referrals to physicians.

As primary care moves to value-based payment, the need for multidisciplinary, team-based care and care delivery by non-physician providers will be necessary to deliver high value care. ${ }^{3}$ Further studies are needed to understand the reasons for potential underuse or overuse of PT referral and to explore new models of care delivery that promote the appropriate use of physical therapists on primary care teams.

Corresponding Author: Janet K. Freburger, PT, PhD; Department of Physical Therapy, School of Health and Rehabilitation Science University of Pittsburgh, Pittsburgh, PA, USA (e-mail: janet_ freburger@pitt.edu).
Funding Information The research reported in this manuscript is supported by a grant awarded through the Health Resources and Services Administration (HRSA) of the U.S. Department of Health and Human Services (HHS) under Cooperative Agreement U81HP2649504-00, Health Workforce Research Centers Program.

\section{Compliance with Ethical Standards:}

Conflict of Interest: The authors declare that they do not have a conflict of interest.

Disclaimer: The funder/sponsor did not play a role in the design and conduct of the study; collection, management, analysis, and interpretation of the data; preparation, review, or approval of the manuscript; and decision to submit the manuscript for publication. The opinions expressed in this manuscript are those of the authors and do not represent the opinions of the sponsor.

\section{REFERENCES}

1. United States Bone and Joint Initiative (USBJI). The burden of musculoskeletal diseases in the United States (BMUS). 3rd ed. Rosemont: USBJI; 2014.

2. Dziedzic KS, French S, Davis AM, Geelhoed E, Porcheret M. Implementation of musculoskeletal models of care in primary care settings: Theory, practice, evaluation and outcomes for musculoskeletal health in highincome economies. Best Pract Res Clin Rheumatol. 2016;30(3):375-97.

3. Ellner AL, Phillips RS. The Coming Primary Care Revolution. J Gen Intern Med. 2017;32(4):380-6.

4. Stata Corp. Stata Survey Data Reference Manual: Release 14. College Station,: Stata Press; 2014.

5. National Center for Health Statistics, Ambulatory Care Statistics Branch. 2012 NAMCS Micro data file documentation. Hyattsville, MD: National Center for Health Statistics; 2012.

6. Williams R. Using the margins command to estimate and interpret adjusted predictions and marginal effects. The Stata Journal. 2012;12(2):308-31. 